\title{
DiVERSIDAD DE HELECHOS Y LICÓFITOS EN FRAGMENTOS DE SELVA MEDIANA SUBPERENNIFOLIA DEL SUR DE TABASCO, MÉXICO
}

\author{
William Cetzal-Ix ${ }^{1,3}$, Eliana Noguera-Savelli², Miguel Martínez-Icó² \\ y Neptalí Ramírez-MarCial ${ }^{2}$ \\ 'El Colegio de la Frontera Sur (ECOSUR), Unidad Chetumal, Chetumal, Quintana Roo, México \\ ${ }^{2}$ Departamento de Conservación de la Biodiversidad, \\ El Colegio de la Frontera Sur (ECOSUR), San Cristóbal de Las Casas, Chiapas, México \\ ${ }^{3}$ Autor para la correspondencia: rolito22@hotmail.com
}

\begin{abstract}
Resumen: A pesar de que más del 90\% del territorio de Tabasco se encuentra deforestado, se registraron 48 taxa de helechos y licófitos en unos pocos fragmentos de selva mediana subperennifolia en Tacotalpa. Estos corresponden a 43 especies, una variedad, una forma de helechos y tres especies de licófitos, agrupadas en 28 géneros y 17 familias. Esta riqueza, representa el $36 \%$ de los taxa, $57 \%$ de los géneros y $63 \%$ de las familias de la flora de helechos y licófitos de Tabasco. Se encontraron ocho nuevos registros para el estado (siete helechos y un licófito). Estas adiciones incrementan la pteridoflora de Tabasco a 124 especies, una subespecie, seis variedades y dos formas. Los géneros con el mayor número de especies son Adiantum (5), Asplenium (5) y Thelypteris (4). En lo que respecta a la forma de vida, 22 especies y una forma son terrestres, siete especies y una variedad epífitas, 12 especies terrestres o epipétricas, dos especies epífitas o epipétricas y tres escandentes. La localidad con el mayor número de especies es La Pila (34), seguido por Tomás Garrido (23), Oxolotán (19) y Grutas de Kolem Chen (15). El mayor número de especies se observó en el interior de los fragmentos de selva mediana subperennifolia (45), seguida de los márgenes de los fragmentos de selva mediana subperennifolia (33) y la vegetación secundaria (17). Por otro lado, 14 especies se observaron exclusivamente en el interior de los fragmentos de selva mediana subperennifolia y una especie en los márgenes de los fragmentos de selva mediana subperennifolia. Además, 30 especies están presentes tanto en el interior como en los márgenes de los fragmentos de selva mediana subperennifolia y 14 especies en los tres tipos de vegetación. La riqueza de las especies evidencia la importancia de estos escasos fragmentos de selva mediana subperennifolia para la supervivencia de los helechos y licófitos en Tabasco.
\end{abstract}

Palabras clave: Adiantum, Asplenium, florística, Lycopodiidae, Polypodiidae, Tacotalpa.

\begin{abstract}
Although over 90\% of the territory of Tabasco is deforested, there were 48 taxa of ferns and lycophytes in a few semiervergreen forest fragments in Tacotalpa. These correspond to 43 species, one variety, and one form of ferns, and three species of lycophytes, grouped in 28 genera and 17 families. This richness represents $36 \%$ of the taxa, $57 \%$ of genera, and $63 \%$ of the families of the flora of ferns and lycophytes of Tabasco. We found eight new records for the state (seven ferns and one lycophyte). These additions increase the pteridoflora of Tabasco to 124 species, one subspecies, six varieties, and two forms. The genera with the largest number of species are Adiantum (5), Asplenium (5), and Thelypteris (4). As regards to life form, 22 species and one form are terrestrial, seven species and one variety are epiphytic, 12 species are terrestrial or epipetric, two species are epiphytic or epipetric, and three species are scandent. The locality with the largest number of species is La Pila (34), followed by Tomás Garrido (23), Oxolotán (19), and Grutas de Kolem Chen (15). The greatest number of species was observed in the interior of the semievergreen forest fragments (45), followed by the margins of the semievergreen forest fragments (33), and secondary vegetation (17). On the other hand, 14 species were observed only in the interior of the semievergreen forest fragments and one species on the margins of the semievergreen forest fragments. Furthermore, 30 species are present both at the interior and margins of the semievergreen forest fragments and 14 species in the three vegetation types. The species richness evidences the importance of these scarce fragments of semievergreen forest as effective refuge for the ferns and lycophytes.
\end{abstract}

Key words: Adiantum, Asplenium, floristics, Lycopodiidae, Polypodiidae, Tacotalpa.

E 1 reconocimiento de los patrones de distribución de la riqueza de especies en ambientes fragmentados es fundamental para entender los efectos del cambio de uso del suelo sobre la diversidad biológica. En gran parte del sureste de
México, la deforestación, la ganadería extensiva, la intensidad agrícola, la urbanización y la explotación petrolera son actividades que han modificado la mayoría de los ecosistemas, principalmente en Tabasco (Sánchez y Barba, 2005). 
En 1950 las selvas de Tabasco ocupaban una extensión de 538,861 ha (21.7\% del territorio estatal). Sin embargo, en las últimas cinco décadas se redujo a 41,079.3 ha (1.65\%), debido a la deforestación por actividades ganaderas y agrícolas (Sánchez-Munguía, 2005).

La deforestación de las selvas no sólo representa una amenaza para la biodiversidad, sino consecuencias a escala local, regional y global, tales como el deterioro de los servicios ambientales (erosión del suelo, alteración de los ciclos del agua y carbón) mediante la perturbación del hábitat y del aislamiento de los fragmentos de selva entre sí (Guevara et al., 2004). En Tabasco, los últimos fragmentos de selva mediana subperennifolia se localizan en la zona serrana en los municipios de Huimanguillo, Macuspana, Tacotalpa, Teapa y Tenosique (Castillo y Zavala, 1996). En estos fragmentos de vegetación existe una alta biodiversidad; se han registrado alrededor de 2,589 especies de plantas (Pérez et al., 2005). Uno de los grupos taxonómicos menos estudiados en esta región es el de los helechos y licófitos, que aunque son favorecidos por los altos niveles de humedad y sombra que le proporcionan los árboles, poco se sabe sobre su distribución en ambientes altamente fragmentados. Los helechos y licófitos pueden ser considerados como indicadores de cambios ambientales debido a su sensibilidad a variaciones microclimáticas (Beukema y van Noorwijk, 2004; Kluge et al., 2006). Sin embargo, la protección de estas especies vegetales no puede darse en forma aislada, su conservación necesariamente tiene que ver con la preservación de las comunidades de las cuales forma parte (Pérez et al., 2005).

Las primeras recolecciones de helechos y licófitos en Tabasco se realizaron en Huimanguillo, Macuspana y Teapa por Ghiesbreght en 1862-1865 y posteriormente por Linden en 1890 (Rovirosa, 1909). El primer estudio formal de los helechos de Tabasco fue realizado por el botánico tabasqueño José Rovirosa (1909), y presentado en su obra Pteridografía del sur de México. Posteriormente Cowan (1983) incluyó los helechos y licófitos en la Flora de Tabasco. En estudios recientes, como los realizados por Magaña-Alejandro (1992), Mickel y Smith (2004) y Pérez et al. (2005), se han registrado diferentes números de especies de helechos y licófitos para el estado (Cuadro 1). Hasta el momento, no hay consenso en cuanto al número de especies; además, todos estos trabajos están basados principalmente en las co- lecciones de Rovirosa y de Cowan, lo que indica que no se han realizado nuevas recolectas y actualizaciones taxonómicas. Los helechos y licófitos en Tabasco representan un grupo de interés histórico, debido a que Rovirosa (1909) fue el primer botánico que se enfocó a estudiarlos para el estado y, posterior a su trabajo, han sido poco recolectados y estudiados (Magaña-Alejandro, 1992). El objetivo de este estudio fue inventariar los helechos y licófitos presentes en los fragmentos de selva mediana subperennifolia para contribuir al conocimiento de la flora de Tabasco.

\section{Materiales y métodos}

Área de estudio. Las localidades de estudio de la cuenca media del río Grijalva en la porción montañosa de Tacotalpa, Tabasco, fueron identificadas con imágenes de satélite y posteriormente corroboradas por el grupo de trabajo de ECOSUR durante la realización de inventarios forestales dentro del proyecto "Gestión y estrategias de manejo sustentable para el desarrollo regional en la cuenca hidrográfica transfronteriza Grijalva”. El área de estudio comprende fragmentos de selva mediana subperennifolia y vegetación secundaria de acuerdo al sistema de clasificación del Instituto Nacional de Estadística y Geografía (INEGI, 2011). Los fragmentos de selva mediana subperennifolia fueron seleccionados con base en su grado de conservación y que no presentasen cultivos en su interior. Las localidades muestreadas fueron las Grutas de Kolem Chen, La Pila, Oxolotán y Tomás Garrido (Figura 1, Cuadro 2).

Muestreos y recolectas botánicas. En los fragmentos de selva mediana subperennifolia se realizaron 24 parcelas circulares de $1,000 \mathrm{~m}^{2}$ utilizadas para inventarios forestales (sensu Ramírez-Marcial et al., 2001). En las localidades Grutas de Kolem Chen y La Pila se establecieron cuatro parcelas en cada una, y en Oxolotán y Tomás Garrido ocho parcelas en cada una (Cuadro 2). En el interior de las parcelas y a lo largo de los caminos de los márgenes de los fragmentos de selva mediana subperennifolia y de la vegetación secundaria se hicieron recolecciones de helechos y licófitos. Se identificaron cuatro formas de vida según la clasificación de Whittaker (1975): epífitas, terrestres, epipétricas y escandentes. La recolecta y procesamiento de los ejemplares

Cuadro 1. Comparación de taxa de helechos y licófitos incluidos por diferentes autores para Tabasco.

\begin{tabular}{|c|c|c|c|c|c|}
\hline Autores & Especies & Subespecies & Variedades & Formas & Det. género \\
\hline Rovirosa (1909) & 50 & - & 5 & - & - \\
\hline Cowan (1983) & 84 & - & - & - & 5 \\
\hline Magaña-Alejandro (1995) & 116 & - & 2 & - & - \\
\hline Mickel y Smith (2004) & 112 & - & 3 & 4 & - \\
\hline Pérez et al. (2005) & 85 & - & 1 & - & - \\
\hline Este estudio & 124 & 1 & 6 & 2 & - \\
\hline
\end{tabular}




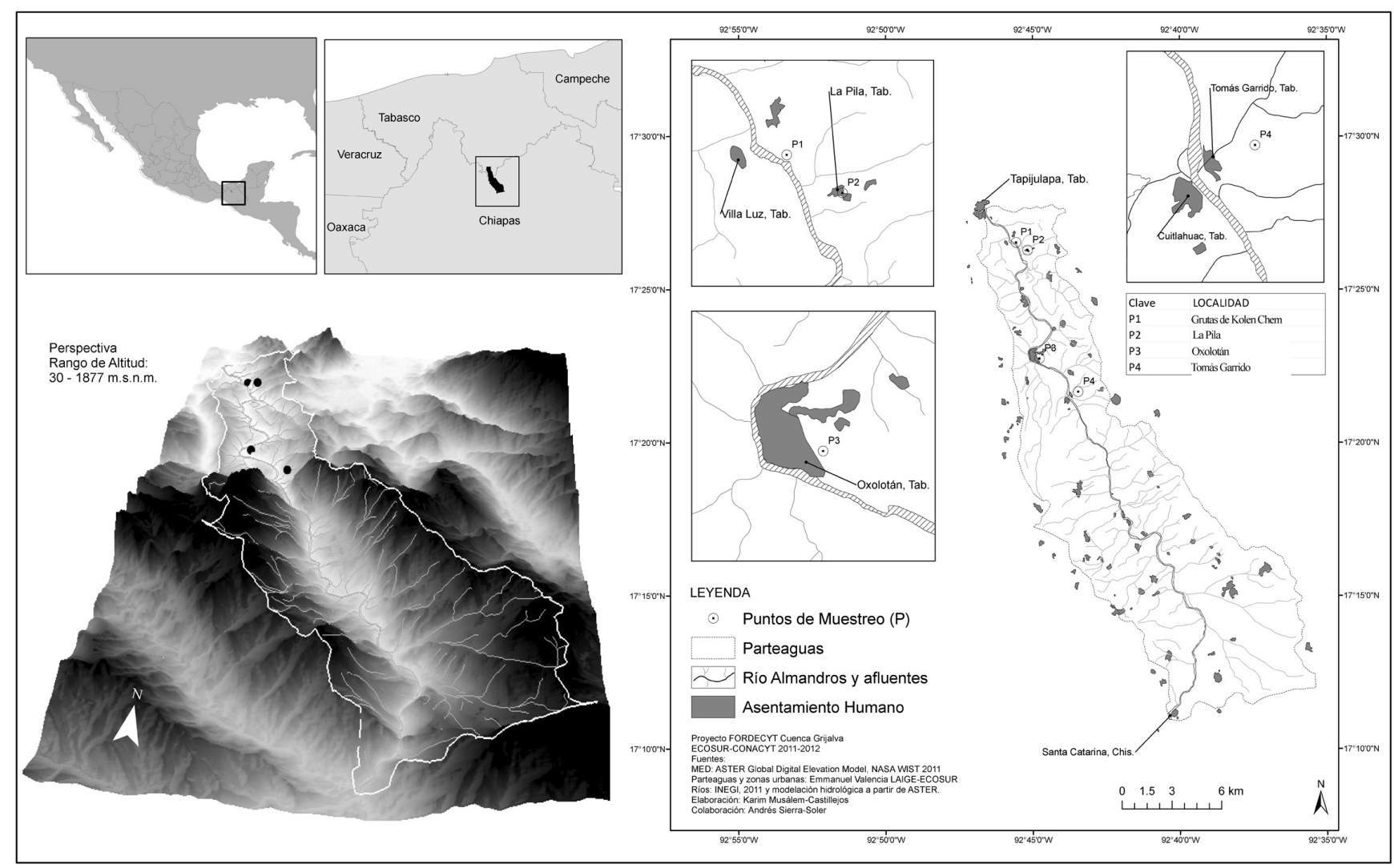

Figura 1. Área de estudio y localidades muestreadas.

botánicos se realizó de acuerdo con la propuesta de Lot y Chiang (1986).

Determinación taxonómica. Las determinaciones botánicas se realizaron con base en el trabajo de Mickel y Smith (2004) y las claves taxonómicas de Flora Mesoamericana (Davidse et al., 1995). Los ejemplares de respaldo se depositaron en los herbarios de El Colegio de la Frontera Sur $(\mathrm{CH})$ y el Centro de Investigación Científica de Yucatán A.C. (CICY). Los taxa fueron ordenados de acuerdo al sistema de clasificación de Christenhusz et al. (2011).

\section{Resultados}

Se registraron 48 taxa repartidos en 43 especies, una variedad y una forma de helechos y tres especies de licófitos, agrupadas en 28 géneros y 17 familias que representan el $36 \%$ de los taxa, $57 \%$ de los géneros y $63 \%$ de las familias de la flora de helechos y licófitos de Tabasco. Los géneros con mayor diversidad de especies son Adiantum (5), Asplenium (5) y Thelypteris (4). Las familias con mayor número de taxa son Pteridaceae (13), Aspleniaceae, Polypodiaceae (5 spp. cada una) y Thelypteridaceae (4).

En lo que respecta a las formas de vida, 22 especies y una forma son terrestres, siete especies y una variedad son epífi- tas, 12 son terrestres o epipétricas, dos especies son epífitas o epipétricas (facultativas) y tres escandentes. En cuanto al número de especies por categoría de vegetación, se registraron 45 especies en el interior de las parcelas, seguido de 33 especies en los márgenes de los fragmentos de selva mediana subperennifolia y 17 especies en la vegetación secundaria. Catorce especies se observaron exclusivamente en el interior de los fragmentos de selva mediana subperennifolia y una especie se registró en los márgenes de los fragmentos de selva mediana subperennifolia; treinta especies se encuentran en el interior y los márgenes de los fragmentos de selva mediana subperennifolia y 14 especies se encuentran en las tres categorías de vegetación (Apéndice).

El número de especies por localidad fue mayor en La Pila con 35 (72\%), seguido por Tomás Garrido con 23 (48\%), Oxolotán con 20 (42\%) y Grutas de Kolem Chen con 15 (31\%). Ocho especies son nuevos registros para Tabasco, cuatro se encontraron en La Pila (Ananthacorus angustifolius, Asplenium miradorense, Blechnum polypodioides y Megalastrum lunense), dos en La Pila y Oxolotán (Huperzia reflexa y Megalastrum atrogriseum), uno en Oxolotán (Nephrolepis pectinata) y uno en Tomás Garrido (Cheilanthes fimbriata). Cuatro de los nuevos registros se observaron exclusivamente en el interior de los fragmentos de selva mediana subperennifolia, tres en el interior y los márgenes de 
William CetZAL-Ix ET AL.

Cuadro 2. Descripción topográfica y de la altura de la vegetación de las localidades estudias.

\begin{tabular}{|c|c|c|c|c|c|}
\hline Localidad & Parcela & $\begin{array}{l}\text { Altitud } \\
\text { (msnm) }\end{array}$ & Orientación & Pendiente & $\begin{array}{l}\text { Altura de los } \\
\text { árboles (m) }\end{array}$ \\
\hline \multirow[t]{4}{*}{ Grutas de Kolem Chen } & 1 & 160 & norte-sur & $42 \% 23^{\circ}$ & 25 \\
\hline & 2 & 140 & noroeste-este & $35 \% 19^{\circ}$ & 30 \\
\hline & 3 & 70 & noroeste-este & $21 \% 12^{\circ}$ & $20-25$ \\
\hline & 4 & 120 & - & $27 \% 15^{\circ}$ & $20-25$ \\
\hline \multirow[t]{4}{*}{ La Pila } & 1 & 600 & noroeste & $5 \% 3^{\circ}$ & 30 \\
\hline & 2 & 630 & noreste & $60 \% 31^{\circ}$ & $35-45$ \\
\hline & 3 & 450 & este-oeste & $60 \% 31^{\circ}$ & $20-25$ \\
\hline & 4 & 530 & noroeste-suroeste & $41 \% 22^{\circ}$ & $18-20$ \\
\hline \multirow[t]{8}{*}{ Oxolotán } & 1 & 180 & sur-oeste & $88 \% 41^{\circ}$ & 27 \\
\hline & 2 & 90 & noroeste & $70 \% 35^{\circ}$ & $20-22$ \\
\hline & 3 & 110 & sur-oeste & $8 \% 4.5^{\circ}$ & $22-25$ \\
\hline & 4 & 120 & sur-este & $5 \% 3^{\circ}$ & 22 \\
\hline & 5 & 110 & sur-este & $52 \% 27.5^{\circ}$ & 20 \\
\hline & 6 & 90 & noreste & $61 \% 31.3^{\circ}$ & $25-27$ \\
\hline & 7 & 80 & sureste & $0 \% 0^{\circ}$ & 25 \\
\hline & 8 & 60 & suroeste & $20 \% 11.5^{\circ}$ & 25 \\
\hline \multirow[t]{8}{*}{ Tomás Garrido } & 1 & 200 & noroeste & $0 \% 30^{\circ}$ & 15 \\
\hline & 2 & 190 & noroeste & $84 \% 40^{\circ}$ & 20 \\
\hline & 3 & 130 & suroeste & $0 \% 40^{\circ}$ & 15 \\
\hline & 4 & 120 & suroeste & $28 \% 55^{\circ}$ & 22 \\
\hline & 5 & 110 & oeste & $24 \% 10^{\circ}$ & 25 \\
\hline & 6 & 90 & - & $1 \% 0.5^{\circ}$ & 20 \\
\hline & 7 & 130 & este-oeste & $58 \% 30^{\circ}$ & 25 \\
\hline & 8 & 150 & suroeste & $41 \% 22.5^{\circ}$ & 15 \\
\hline
\end{tabular}

los fragmentos de selva mediana subperennifolia y uno en las tres categorías de vegetación (Cuadro 2).

\section{Discusión}

Riqueza de especies. Los fragmentos estudiados de selva mediana subperennifolia se encuentran en la porción serrana del sur de Tabasco a una altura de entre 50 a $630 \mathrm{~m}$ y forman parte de los márgenes de las montañas del norte de Chiapas y de una zona denominada "área del arco" que es una región continua, de alta precipitación (3,000 mm y en algunas partes mayor a 5,000 mm) que inicia en Uxpanapa, Veracruz, y termina en las montañas del sur de Tabasco (Wendt, 1989). Por otra parte, en esta porción serrana suceden escurrimientos que descienden de las montañas del norte de Chiapas (Ortíz-Pérez et al., 2005), que crean condiciones de humedad a lo largo de la cuenca media del río Grijalva, por lo que no es casualidad encontrar esta alta riqueza de helechos y licófitos (20 spp/ha).

Lo anterior coincide por lo expuesto por Mehltreter (2008), que la mayor riqueza de helechos se encuentra a los $1,000 \mathrm{~m}$ o un poco inferior. En las selvas altas perennifolias la diversidad local puede alcanzar desde $50 \mathrm{spp} / \mathrm{ha}$ en Ecuador hasta 2,100 spp/ha en Costa Rica. Sin embargo, los helechos son más diversos a elevaciones medias de áreas tropicales montañosas, especialmente donde predominan el bosque mesofilo de montaña o bosque de neblina (Mehltreter, 1995). Mientras a elevaciones bajas la riqueza de especies disminuye, como en la Península de Yucatán (Campeche, Quintana Roo y Yucatán) con vegetación predominante de selva mediana subperennifolia, subcaducifolia y selva baja caducifolia, los helechos y licófitos están representados por 65 especies en 32 géneros y 15 familias; esta baja diversidad se explica porque son plantas altamente dependientes de agua y humedad ambiental y, la existencia de un largo período de sequía en casi toda la Península de Yucatán hace de la mayoría de sus hábitats ambientes poco apropiados para sus requerimientos ecológicos (Carnevali et al., 2010).

Los helechos y licófitos están afectados por el cambio del uso del suelo que reduce la disponibilidad de hábitats adecuados para su supervivencia (Mehltreter, 2008), pero por otra lado favorece a especies de ambientes secundarios que crecen a orillas de bosque (Megalastrum atrogriseum y $M$. lunense), a lo largo de los caminos (Tectaria heracleifolia), en taludes (Pteris grandifolia y P. longifolia) o a orillas de vegetación secundaria (Lygodium heterodoum y L. venustum), aunque también fueron observadas en el interior de los fragmentos de selva mediana subperennifolia. Las es- 

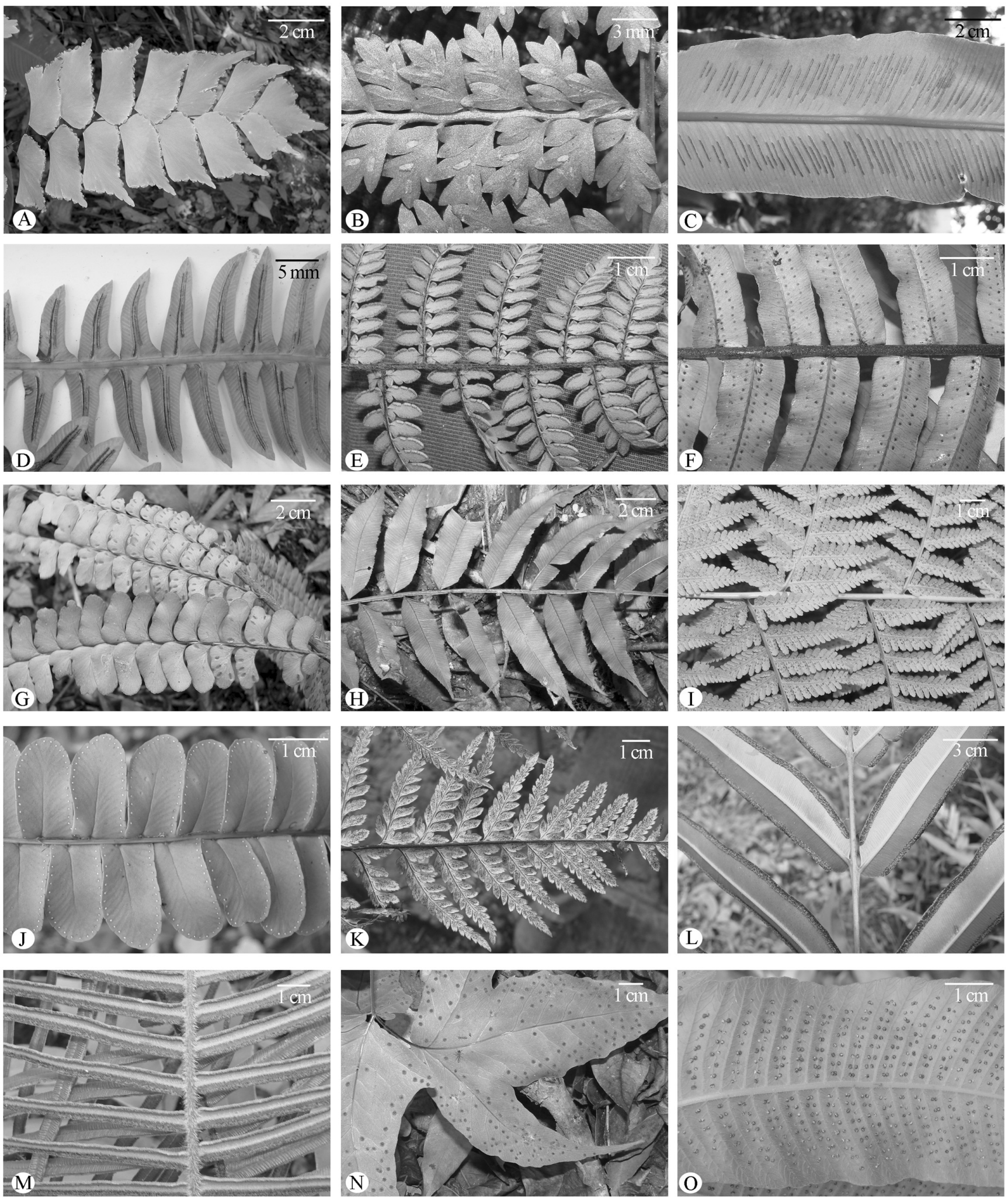

Figura 2. Helechos. A. Adiantum trapeziforme. B. Asplenium cristatum. C. Asplenium serratum. D. Blechnum polypodioides. E. Cheilanthes fimbriata. F. Cyclopeltis semicordata. G. Didymochlaena truncatula. H. Lomariopsis mexicana. I. Megalastrum atrogriseum. J. Nephrolepis pectinata. K. Pityrogramma calomelanos. L. Pteris grandifolia. M. Pteris longifolia. N. Tectaria heracleifolia. O. Thelypteris ghiesbreghtii. 

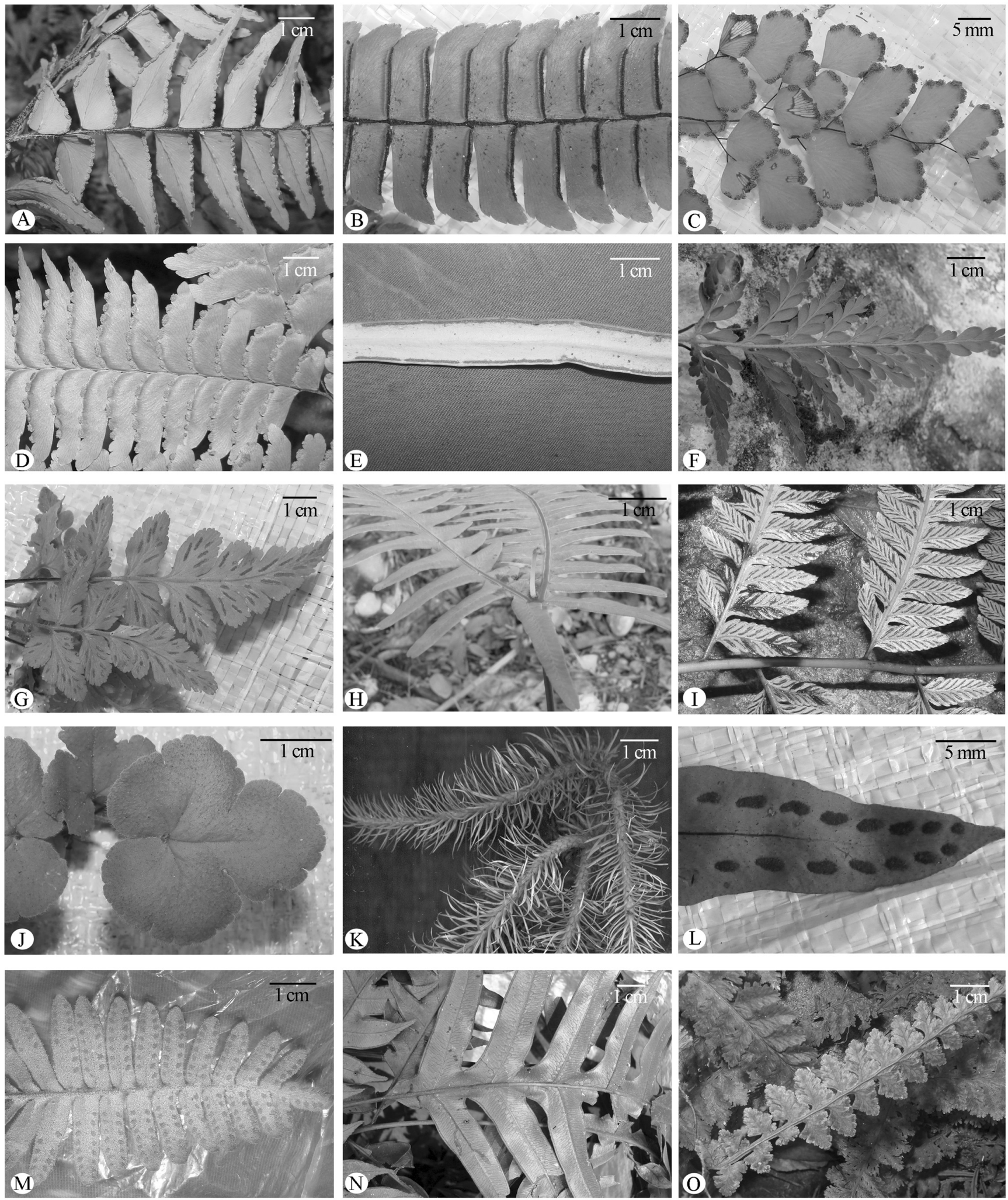

Figura 3. Helechos y licófitos. A. Adiantum latifolium. B. Adiantum pulverulentum. C. Adiantum tricholepis. D. Adiantum wilesianum. E. Ananthacorus angustifolius. F. Anemia adiantifolia. G. Asplenium pumilum. H. Dicranopteris flexuosa. I. Diplazium lonchophyllum. J. Hemionitis palmata. K. Huperzia reflexa. L. Pleopeltis astrolepis. M. Pleopeltis polypodioides var. polypodioides. N. Pteris altissima. O. Vandenboschia radicans. 
pecies anteriores no presentan problemas de supervivencia $\mathrm{y}$, por lo general, son consideradas como colonizadoras o invasoras de ambientes perturbados (Tejero-Díez, 2007). Sin embargo, un buen porcentaje de los helechos y licófitos (29\%), en este estudio, se observaron únicamente en el interior de los fragmentos de selva mediana subperennifolia, creciendo como epífitas (p. ej., Ananthacorus angustifolius, Huperzia reflexa, Vandenboschia radicans y Vittaria lineata) o terrestres (p. ej., Adiantum trapeziforme, Asplenium abscissum, A. cristatum, A. miradorense, Campyloneurum angustifolium, Diplazium lonchophyllum, Didymochlaena truncatula, Pteris altissima y P. orizabae). Las especies epífitas son altamente dependiente de los árboles que son sus hospederos, mientras que las especies facultativas (epífitas o terrestres) únicamente logran sobrevivir en el sotobosque del interior de los fragmentos de vegetación. En este contexto los fragmentos de selva mediana pueden representar los últimos reservorios en Tabasco para las especies no tolerantes a condiciones de perturbación, como el caso de algunos de los nuevos registros (Anantachorus angustifolius, Asplenium miradorense, Huperzia reflexa y Megalastrum lunense) que se encuentran exclusivamente en este tipo de vegetación.

La contribución de los helechos y licófitos al total de la riqueza de taxa para el estado representa el $36 \%$ e incrementa el número a 124 especies, una subespecie, seis variedades y dos formas. La localidad de La Pila contiene el mayor número de especies ( $85 \mathrm{spp} / \mathrm{ha})$ y fue donde se encontraron cuatro de los ocho nuevos registros. La riqueza de especies de esta parcela está asociada a que fue un sitio aislado de los poblados, de difícil acceso y mejor conservado, con árboles de 18 a $35 \mathrm{~m}$ de altura, y ubicada a mayor altitud, entre 450 a $630 \mathrm{~m}$. Tomás Garrido fue la segunda localidad con mayor número de especies ( $29 \mathrm{spp} / \mathrm{ha}$ ), con árboles de 15 a $25 \mathrm{~m}$ de altura, y las parcelas ubicadas entre 90 a $200 \mathrm{~m}$ de altitud. Oxolotán (24 spp/ha) con árboles de 20 a $27 \mathrm{~m}$ de altura, y las parcelas ubicadas entre 60 a $180 \mathrm{~m}$ de altitud, con sustrato rocoso calcáreo. En esta localidad las especies más abundantes en el interior de los fragmentos fueron Cyclopeltis semicordata y Tectaria heracleifolia, y en los taludes de los márgenes de los fragmentos de vegetación Pteris grandifolia y P. longifolia. Estas dos últimas especies de amplia distribución han sido usualmente registradas en los márgenes de vegetación y suelos rocosos (Mickel y Smith, 2004). En las parcelas de las Grutas de Kolem Chen se registraron el menor número de especies (38 spp/ha), las cuales se encuentran cercanas a La Pila y la vegetación está también conservada, los árboles presentan altura de 20 a $30 \mathrm{~m}$; sin embargo, se encuentran a una altitud más baja, de entre 70 a $160 \mathrm{~m}$ y con un sustrato rocoso calcáreo; en esta localidad las especies con mayor abundancia fueron Cyclopeltis semicordata y Tectaria heracleifolia, ambas especies epipétricas y de amplia distribución. En todas las localidades se registró la presencia de Cyclopeltis semicordata, Tectaria hera- cleifolia y Thelypteris tetragona, especies de amplia distribución que se encuentran desde el nivel del mar hasta los 1,000 m de altura y en diversos tipos de vegetación (Mickel y Smith, 2004); por lo que no fue extraño encontrarlas en todas localidades y categorías de vegetación.

Nuevos registros. De los nuevos registros, Ananthacorus angustifolius y Blechnum polypodioides presentan amplia distribución en México. Blechnum polypodioides crece como terrestre o epífita en lugares abiertos o bosques de pino encino a elevaciones desde $50 \mathrm{~m}$ hasta los $1,450(-3,000) \mathrm{m}$ (Mickel y Smith, 2004). En La Pila se encontró como especie terrestre en un talud de los márgenes de los fragmentos de selva mediana subperennifolia, a una elevación de 450 $\mathrm{m}$. Ananthacorus angustifolius crece como epífita o epipétrica en selva alta, mediana y baja perennifolia desde 50 hasta 1,500 m (Mickel y Smith, 2004), y recientemente fue registrada por Carnevali et al. (2010) para Quintana Roo en selva mediana subperennifolia. Esta especie fue encontrada en La Pila, creciendo como epífita únicamente en el interior de los fragmentos de selva mediana subperennifolia a una altitud de $530 \mathrm{~m}$.

Tres de los nuevos registros, Asplenium miradorense, Huperzia reflexa y Nephrolepis pectinata se distribuyen en México en la porción central y suroeste del país, incluyendo la porción norte de Chiapas en el límite con Tabasco; sin embargo, no habían sido recolectadas para Tabasco. $\mathrm{Hu}$ perzia reflexa crece como terrestre o epífita en selva altas, medianas y bajas perennifolias a elevaciones de 300 a 2,400 $\mathrm{m}$ (Mickel y Smith, 2004). En el presente estudio, esta especie se encontró en La Pila y Oxolotán, creciendo como epífita únicamente en el interior de los fragmentos de selva mediana subperennifolia a altitudes de 200 y 600 m, respectivamente. Por otra parte, Asplenium miradorense ha sido registrada como epífita desde bosque de pino-encino, bosque mesófilo hasta selvas altas perennifolias a elevaciones de (500-)1,000 a 2,100 (-2,500) m (Davidse et al., 1995); esta especie se encontró en La Pila como terrestre en el interior de la selva mediana subperennifolia a una altitud de 630 m. Asimismo, Nephrolepis pectinata crece como terrestre o epipétrica, desde bosque de pino-encino, bosque mesófilos hasta selvas altas perennifolias a elevaciones de 150 a 1,200 $(-1,700) \mathrm{m}$, en este estudio se encontró en Oxolotán únicamente en el interior de los fragmentos de selva subperennifolia a una altitud de $180 \mathrm{~m}$.

Dos de los nuevos registros, Cheilanthes fimbriata y Megalastrum atrogriseum se distribuyen en México en la vertiente atlántica en algunos estados del Golfo; sin embargo, no habían sido recolectados para Tabasco. Megalastrum atrogriseum se conocía previamente de Veracruz y Oaxaca creciendo en selvas altas y medianas perennifolias a elevaciones de (100-) 250 a $500(-2,000) \mathrm{m}$; en este estudio se encontró en La Pila y Oxolotán como especie terrestre, en selva mediana subperennifolia a una altitud de $450 \mathrm{~m}$ a 
$180 \mathrm{~m}$, respectivamente. Por otro lado, Cheilanthes fimbria$t a$ crece en áreas abiertas de selva mediana subperennifolia, desde el nivel del mar hasta los 1,000 m. En este estudio fue encontrada en Tomás Garrido como especie terrestre en el interior y los márgenes de los fragmentos de la selva mediana subperennifolia y en la vegetación secundaria, a una altitud de 90 a $200 \mathrm{~m}$.

En el caso de Megalastrum lunense, se conocía en México únicamente del sureste de Chiapas en bosque húmedos a elevaciones de (600-) 800 a 1,000 (-1,800) m (Mickel y Smith, 2004); en este estudio fue encontrada en La Pila como especie terrestre en el interior y los márgenes de los fragmentos de selva mediana subperennifolia a una altitud de $630 \mathrm{~m}$.

De los ocho nuevos registros mencionados para Tabasco, cuatro fueron encontrados únicamente en el interior de los fragmentos de selva mediana subperennifolia, pero con los ritmos actuales de deforestación es probable que en poco tiempo desaparezcan estas últimas reminiscencias de vegetación selvática con lógicas consecuencias en la modificación del microambiente local. Estas tendencias ya habían sido anticipadas desde hace varias décadas atrás (p. ej., Eckholm, 1975).

A pesar de que se han realizado varios inventarios florísticos en diversas zonas de Tabasco, el hallazgo de los ocho nuevos registros de helechos y licófitos aquí presentados, evidenció la necesidad de continuar los estudios florísticos en otras áreas poco estudiadas del estado que aún poseen remanentes de vegetación selvática conservada.

\section{Agradecimientos}

A Mario González Espinosa (ECOSUR) por todas las facilidades otorgadas para el desarrollo y culminación del presente trabajo de investigación. Trabajo de campo financiado por el proyecto FORDECYT 143303 "Gestión y estrategias de manejo sustentable para el desarrollo regional en la cuenca hidrográfica transfronteriza Grijalva" y por la Secretaría de Recursos Naturales y protección Ambiental del estado de Tabasco (SERNAPAM). El primer autor agradece al CONACYT por la beca 162579 otorgada para estudios doctorales. La segunda autora agradece al ECOSUR por la beca otorgada para estudios posdoctorales. A los curadores de los herbarios CH y CICY por su colaboración por la recepción del material herborizado. A Alfonso Luna, Jesús Chi Quej, Soledad Gómez Gómez, Zenaida Hernández López, Olmar Santis López y Henry Castañeda (ECOSUR) por su colaboración durante las salidas de campo. A Karim Musálem Castillejos (ECOSUR) por la elaboración de la figura 1. A Germán Carnevali (CICY) por sus comentarios al manuscrito. Agradecemos los comentarios y sugerencias de un árbitro anónimo. Agradecemos especialmente a Klaus Mehltreter por sus valiosos comentarios, sugerencias e identificaciones que ayudaron a mejorar en gran medida este manuscrito.

\section{Literatura citada}

Beukema H. y van Noordwijk M. 2004. Terrestrial pteridophytes as indicators of a forest-like environment in rubber production systems in the lowlands of Jambi, Sumatra. Agriculture, Ecosystems and Environment 104:63-73.

Carnevali F.C.G., Tapia-Muñoz J.L., Duno de Stefano R. y Ramírez M.I. 2010. Flora Ilustrada de la Península de Yucatán: Listado Florístico. Centro de Investigación Científica de Yucatán, A. C., Mérida.

Castillo A.O. y Zavala C.J. 1996. Fisiografía, recursos vegetales y alternativas de manejo en el Parque Estatal Agua Blanca Tabasco. Universidad y Ciencia 12:63-70.

Christenhusz M.J.M., Xian-Chun Z. y Scheneider H. 2011. A linear sequence of extant families and genera of lycophytes and ferns. Phytotaxa 19:7-54.

Cowan C.P. 1983. Listados Florísticos de México. I. Flora de Tabasco. Instituto de Biología, Universidad Nacional Autónoma de México, México, D.F.

Davidse G., Sousa S.M. y Knapp S. 1995. Flora Mesoamericana. Volumen 1: Psilotaceae a Salvinaceae. Universidad Nacional Autónoma de México, Instituto de Biología, Missouri Botanical Garden, The Natural History Museum, México, D.F.

Eckholm E.P. 1975. The deterioration of mountain environments. Science 189:764-770.

Guevara S.S., Sánchez-Ríos G. y Landgrave R.R. 2004. La deforestación. En: Guevara S., Laborde, J. y Sánchez-Ríos. G. Eds. Los Tuxtlas, El Paisaje de la Sierra, pp. 85-108, Instituto de Ecología, A.C., Unión Europea, Xalapa.

INEGI. Instituto Nacional de Estadística y Geografía. 2011. Vegetación de Tabasco. <http://mapserver.inegi.gob.mx/geografia/espanol/estados/tab/agri.cfm?c $=444 \&$ e $=07>($ consultado 10 junio 2012).

Kluge J., Kessler M. y Dunn R.R. 2006. What drives elevational patterns of diversity? A test of geometric constraints, climate and species pool effects for pteridophytes on an elevational gradient in Costa Rica. Global Ecology and Biogeography 15:358-371.

Lot A. y Chiang F. 1986. Manual de Herbario. Administración y Manejo de Colecciones, Técnicas de Recolección y Preparación de Ejemplares Botánicos. Consejo Nacional de la Flora de México, A.C., México, D.F.

Magaña-Alejandro M.A. 1992. Helechos de Tabasco. Universidad Juárez Autónoma de Tabasco, Centro de Investigación de Ciencias Biológicas, Villahermosa.

Mehltreter K. 1995. Species richness and geographical distribution of montane pteridophytes of Costa Rica, Central America. Feddes Repertorium 106:563-584.

Mehltreter K. 2008. Helechos. En: Manson R.H., Hernández-Ortiz V., Gallina S. y Mehltreter K. Eds. Agroecosistemas Cafetaleros de Veracruz: Biodiversidad, Manejo y Conservación, pp. 83-93, Instituto Nacional de Ecología, A.C., Instituo Nacional de Ecología, México, D.F.

Mickel J.T. y Smith A.R. 2004. The Pteridophytes of Mexico. Memoirs of the New York Botanical Garden 88. The New York Botanical Garden Press, Nueva York.

Ortíz-Pérez M.A., Siebe C. y Cram S. 2005. Diferenciación ecogeográfica de Tabasco. En: Bueno J., Álvarez F. y Santiago S. Eds. Biodiversidad del Estado de Tabasco, pp. 305-322, Instituto de Biología, UNAM-CONABIO, México, D.F. 
Pérez L.A., Sousa S.M., Hanan A.M., Chiang F. y Tenorio P. 2005. Vegetación Terrestre. En: Bueno J., Álvarez F. y Santiago S. Eds. Biodiversidad del Estado de Tabasco, pp. 65-110, Instituto de Biología, UNAM-CONABIO, México, D.F.

Ramírez-Marcial N., González-Espinosa M. y Williams-Linera G. 2001. Anthropogenic disturbance and tree diversity in montane rain forest in Chiapas, Mexico. Forest Ecology and Management 154:311-326.

Rovirosa J.N. 1909. Pteridografía del Sur de México. O sea Clasificación y Descripción de los Helechos de esta Región, Precedida de un Bosquejo de la Flora General. Imprenta de Ignacio Escalante. Edición Facsimilar de la Sociedad Mexicana de Historia Natural (1976), México, D.F.

Sánchez A.J. y Barba E. 2005. Biodiversidad de Tabasco. En: Bueno J., Álvarez F. y Santiago S. Eds. Biodiversidad del Estado de
Tabasco, pp. 1-16, Instituto de Biología, UNAM-CONABIO, México, D.F.

Sánchez-Munguía A. 2005. Uso del Suelo Agropecuario y Deforestación en Tabasco 1950-2000. División Académica de Ciencias Biológicas, Universidad Juárez Autónoma de Tabasco, Villahermosa.

Tejero-Díez J.D. 2007. La riqueza florística del estado de México: licopodios y helechos. Adumbrationes ad Summae Editionem 27:1-32.

Wendt T. 1989. Las selvas de Uxpanapa, Veracruz-Oaxaca, México. Evidencia de refugios florísticos cenozoicos. Anales del Instituto de Biología Universidad Nacional Autónoma de México, Serie Botánica 58:29-54.

Whittaker R.H. 1975. Communities and Ecosystems. MacMillan Publishing, Nueva York.

Recibido: 26 de julio de 2012

Aceptado: 20 de noviembre de 2012 
Apéndice. Lista florística de los fragmentos de selva mediana subperennifolia, Tacotalpa, Tabasco. Colectores: Cetzal \& Noguera $(C \& N)$. Formas de vida: Terrestre (T), Epífita (E), Epipétrica (EP), Escandentes (ES). Tipos de vegetación: Interior del fragmento de selva mediana subperennifolia (IFSM), Margen del fragmento de selva mediana subperennifolia (MFSM), Vegetación secundaria (VS). Presente (P), Ausente (-). Las especies marcadas con asterisco $\left(^{*}\right)$ son nuevos registros para Tabasco. Localidades: Tomás Garrido (T), Grutas de Kolem Chen (K), Oxolotán (O), La Pila (LP). Ausente (-), Presente (P).

\begin{tabular}{llllcc}
\hline Especie y datos de colecta & Tipos de vegetación & Hábito & \multicolumn{2}{c}{ Localidades } \\
& IFSM MFSM VS & T & K & O LP \\
\hline
\end{tabular}

\section{EQUISETOPSIDA}

LYCOPODIIDAE [Licófitos]

LYCOPODIACEAE

Huperzia reflexa (Lam.) Trevis (C\&N 113)*

\section{SELAGINELLACEAE}

Selaginella finitima Mickel \& Beitel (C\&N 82)

Selaginella mickelii Valdespino (C\&N 67)

$\begin{array}{llcllll}\mathrm{P} & - & \mathrm{T} & - & \mathrm{P} & - & - \\ \mathrm{P} & - & \mathrm{T} / \mathrm{P} & - & - & - & \mathrm{P}\end{array}$

\section{POLYPODIIDAE [Helechos]}

ANEMIACEAE

Anemia adiantifolia (L.) Sw. (C\&N 51, 94, 145)

\section{ASPLENIACEAE}

Asplenium abscissum Willd. (C\&N 108, 132)

Asplenium cristatum Lam. (C\&N 65, 73)

Asplenium miradorense Liebm. (C\&N 100) *

Asplenium pumilum Sw. (C\&N 71, 105)

Asplenium serratum L. (C\&N 60, 74, 103, 118)

\section{ATHYRIACEAE}

Diplazium lonchophyllum Kunze (C\&N 45, 109, 147)

\section{BLECHNACEAE}

Blechnum polypodioides Raddi. (C\&N 87, 124)*

\section{DRYOPTERIDACEAE}

Ctenitis salvinii (Baker) Stolze (C\&N 77)

Megalastrum atrogriseum (C. Chr.) A.R.Sm.

\& R.C.Moran (C\&N 137) *

Megalastrum lunense (Christ) A.R.Sm. \& R.C.Moran

$(C \& N$ 89, 90, 138) *

\section{GLEICHENIACEAE}

Dicranopteris flexuosa (Schrad.) Underw. (C\&N 99)

\section{HYMENOPHYLLACEAE}

Vandenboschia radicans (Sw.) Copel. (C\&N 102)

HYPODEMATIACEAE

Didymochlaena truncatula (Sw.) J.Sm. (C\&N 92)
- P P

T/EP

$\mathrm{P} \quad-\quad-\quad-$

$\mathrm{P} \quad-\quad-$

$\mathrm{P} \quad-\quad-$

$\mathrm{P} \quad \mathrm{p} \quad-$

$\mathrm{P} \quad \mathrm{P} \quad-$

T/EP
$\mathrm{T}$
$\mathrm{T}$
$\mathrm{T} / \mathrm{EP}$
$\mathrm{T} / \mathrm{EP}$

T/EP

$\begin{array}{llll}- & P & - & P \\ P & P & - & - \\ - & - & - & P \\ P & P & - & P \\ P & P & - & P\end{array}$

P

$\mathrm{T}$

$\mathrm{P}$

$P$

P

$$
\text { P }
$$$$
-
$$

T

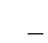

$\mathrm{P}$

$\begin{array}{llll}\mathrm{P} & \mathrm{P} & - & \mathrm{T}\end{array}$

$P$

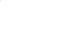

$P$

$-$

T

$\begin{array}{llll}- & \mathrm{P} & - & -\end{array}$

$P$

$-\quad-\quad-\quad P$

\section{$\mathrm{T}$}

E

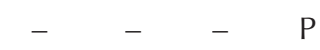


Apéndice. Continuación

Especie y datos de colecta

Tipos de vegetación

IFSM MFSM VS

LOMARIOPSIDACEAE

Cyclopeltis semicordata (Sw.) J.Sm. (C\&N 56, 85, 110, 111, 141)

Lomariopsis mexicana Holttum. (C\&N 47, 72)

Lomariopsis vestita Humb. \& Bonpl. ex Willd. (C\&N 107)

\section{LYGODIACEAE}

Lygodium heterodoxum Kunze (C\&N 57, 81, 112)

Lygodium venustum Sw. (C\&N 116, 131)

\section{NEPHROLEPIDACEAE}

Nephrolepis hirsutula (G.Forst.) C.Presl (C\&N 42, 95)

Nephrolepis pectinata (Willd.) Schott (C\&N 139)*

\section{POLYPODIACEAE}

Campyloneurum angustifolium (Sw.) Fée (C\&N 114, 126, 143)

Microgramma nitida (J.Sm.) A.R.Sm. (C\&N 49)

Pecluma atra (A.M. Evans) M.G. Price (C\&N 43, 115, 123, 154 )

Pleopeltis astrolepis (Liebm.) E.Fourn. (C\&N 48, 96, 125 )

Pleopeltis polypodioides var. polypodioides (L.) E.G.

Andrews \& Windham. (C\&N 63, 86, 155)

PTERIDACEAE

Ananthacorus angustifolius (Sw.) Underw. (C\&N 128, 219) *

Adiantum latifolium Lam. (C\&N 78, 151)

Adiantum pulverulentum L. (C\&N 80, 101, 140, 149)

Adiantum trapeziforme L. (C\&N 97, 122, 134, 150)

Adiantum tricholepis Fée (C\&N 54, 79, 98)

Adiantum wilesianum Hook. (C\&N 64, 152)

Cheilanthes fimbriata (A.R.Sm.) Mickel \& Beitel (C\&N 44)*

Hemionitis palmata L. (C\&N 55, 70, 104)

Pityrogramma calomelanos (L.) Link. (C\&N 66, 127, 135, 148)

Pteris altissima Poir. (C\&N 91, 146)

Pteris grandifolia L. (C\&N 52, 93, 120, 121, 156)

Pteris longifolia L. (C\&N 133, 142, 157)

Vittaria lineata (L.) Sm. (C\&N 50)

\section{TECTARIACEAE}

Tectaria heracleifolia (Willd.) Underw. (C\&N 58, 68, 117, 129)

Tectaria incisa f. incisa Cav. (C\&N 62, 119)

\section{THELYPTERIDACEAE}

Thelypteris ghiesbreghtii (Hook) C.V.Morton (C\&N 61)

Thelypteris hondurensis L.D.Gómez (C\&N 75, 106, 130)

Thelypteris patens (Sw.) Small (C\&N 161)

Thelypteris tetragona (Sw.) Small (C\&N 46, 76, 83, 84, 136, 160)
$P$

$P$

$P$

$P$

$P$

$\mathrm{P}$

$\mathrm{P}$

$\mathrm{P}$

$P$

$P$

$\mathrm{P}$

P

P

$\mathrm{P}$

Hábito

Localidades

$\begin{array}{llll}\text { T } & \text { K } & \text { O } & \text { LP }\end{array}$

$\begin{array}{lllcllll}\mathrm{P} & \mathrm{P} & - & \mathrm{T} & \mathrm{P} & \mathrm{P} & \mathrm{P} & \mathrm{P} \\ \mathrm{P} & - & - & \mathrm{ES} & - & \mathrm{P} & - & - \\ \mathrm{P} & - & - & \mathrm{T} / \mathrm{EP} & - & - & - & \mathrm{P}\end{array}$

$\begin{array}{llllllll}P & P & P & E S & P & P & - & P \\ P & P & P & E S & - & - & - & P\end{array}$

$\begin{array}{llll}P & P & - & E\end{array}$

$\mathrm{T} / \mathrm{EP}$

$\begin{array}{cllll}\text { E/EP } & P & - & P & P \\ E & P & - & - & - \\ \text { E } & P & - & P & P \\ \text { E/EP } & P & - & - & P \\ E & P & - & P & P\end{array}$

$\mathbf{P}$

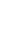

(1)

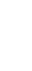


